\begin{tabular}{ll|l}
\cline { 2 - 3 } & \multicolumn{3}{l}{ Intervent Neurol 2016;5:118-122 } \\
\cline { 2 - 3 } & $\begin{array}{l}\text { DOI: 10.1159/000446749 2016 S. Karger AG, Basel } \\
\text { Published online: June 16, } 2016\end{array}$ & $\begin{array}{l}\text { (c) } \\
\text { 1664-9737/16/0054-0118 } \$ 39.50 / 0 \\
\text { www.karger.com/ine }\end{array}$ \\
\hline
\end{tabular}

\title{
Recanalization and Angiographic Reperfusion Are Both Associated with a Favorable Clinical Outcome in the IMS III Trial
}

\author{
Marie L. Schmitz ${ }^{a}$ Sharon D. Yeatts ${ }^{b}$ Thomas A. Tomsick ${ }^{c}$ \\ David S. Liebeskind ${ }^{d} \quad$ Achala Vagal $^{c}$ Joseph P. Broderick ${ }^{c}$ \\ Pooja Khatric for the IMS III investigators \\ a Department of Neurology, Aarhus University Hospital, Aarhus, Denmark; ${ }^{b}$ Medical \\ University of South Carolina (MUSC), Charleston, S.C., ' University of Cincinnati College of \\ Medicine, Cincinnati, Ohio, and dUniversity of California Los Angeles (UCLA), Los Angeles, \\ Calif., USA
}

\section{Key Words}

Acute stroke · Endovascular stroke therapy · Ischemic stroke $\cdot$ Revascularization · Stroke thrombectomy

\begin{abstract}
Background: Prompt revascularization is the main goal of acute ischemic stroke treatment. We examined which revascularization scale - reperfusion (modified Treatment in Cerebral Infarctions, mTICI) or recanalization (Arterial Occlusive Lesion, AOL) - better predicted the clinical outcome in ischemic stroke participants treated with endovascular therapy (EVT). Additionally, we determined the optimal thresholds for the predictive accuracy of each scale. Methods: We included participants from the Interventional Management of Stroke (IMS) III trial with complete occlusion in the internal carotid artery terminus or proximal middle cerebral artery (M1 or M2) who completed EVT within $7 \mathrm{~h}$ of symptom onset. The abilities of the $\mathrm{AOL}$ and $\mathrm{mTICI}$ scales to predict a favorable outcome (defined as a modified Rankin Scale score of $0-2$ at 3 months) were compared by receiver operating characteristic analyses. The maximal sensitivity and specificity for each revascularization scale were established. Results: Among 240 participants who met the study inclusion criteria, 79 (33\%) achieved a favorable outcome. Higher scores of $\mathrm{mTICI}$ and AOL increased the likelihood of a favorable outcome ( $2.7 \%$ with mTICI 0 vs. $83.3 \%$ with mTICI 3 , and $3.0 \%$ with AOL 0 vs. $43 \%$ with AOL 3 ). The accuracy of $\mathrm{mTICI}$ reperfusion and $\mathrm{AOL}$ recanalization for a favorable outcome prediction was similar, with optimal thresholds of $\mathrm{mTICI} 2 \mathrm{~b} / 3$ and AOL 3, respectively. Conclusion: Reperfusion ( $\mathrm{mTICI}$ ) and recanalization (AOL) predicted a favorable clinical outcome with comparable
\end{abstract}

Marie Louise Schmitz, MD

Department of Neurology, Aarhus University Hospital

Norrebrogade 44

DK-8000 Aarhus C (Denmark)

E-Mail mariesch@rm.dk 
accuracy in ischemic stroke participants treated with EVT. Optimal revascularization goals to maximize clinical outcome (modified Rankin Scale score of 0-2) consisted of complete recanalization $(\mathrm{AOL} 3)$ and reperfusion of at least $50 \%$ of the arterial tree of the symptomatic artery $(\mathrm{mTICI} 2 \mathrm{~b} / 3)$ in the IMS III trial setting.

(C) 2016 S. Karger AG, Basel

\section{Introduction}

Early revascularization by restoring blood flow to hypoperfused but still viable brain tissue is crucial for achieving a favorable clinical outcome in patients with acute ischemic stroke [1]. Revascularization is defined as all treatment-related improvements in cerebral blood flow, and current recommendations suggest that recanalization and reperfusion should both be measured when drawing conclusions on the revascularization success [2]. Recanalization measures the direct impact of the medical or mechanical intervention on the most proximal intracranial occlusive lesion causing the stroke symptoms. Hence, recanalization refers to the degree of arterial patency, or clot burden, as graded by the Arterial Occlusive Lesion (AOL) scale, with grade 0 indicating no recanalization of the occlusion and grade 3 equaling complete recanalization of the occlusion with any distal flow [3]. Angiographic reperfusion, on the other hand, refers to restoration of the blood flow to the downstream territory of the symptomatic artery, and thereby depicts the efficacy of acute stroke treatment to restore the blood flow at tissue level. The modified Treatment in Cerebral Ischemia (mTICI) is the preferred measure of angiographic reperfusion, with grade 0 indicating no perfusion and grade 3 representing full perfusion of all visualized distal branches [2].

The recent positive endovascular trials in favor of endovascular therapy (EVT) [4-8] highlight the necessity for additional knowledge on the imaging and clinical characteristics that are associated with treatment success in acute ischemic stroke. Therefore, the aim of our study was to determine which revascularization scale (mTICI vs. AOL), and at which threshold, would best predict a favorable clinical outcome in ischemic stroke participants undergoing EVT.

\section{Methods}

The Interventional Management of Stroke (IMS) III trial did not demonstrate the superiority of the combined approach of intravenous (IV) tissue-plasminogen activator (tPA) and EVT for improving clinical outcomes as compared to IV-tPA alone in ischemic stroke participants [9], although secondary analysis suggested a potential benefit of EVT among participants with occlusions visualized on baseline CTA [10]. Nevertheless, the IMS III trial provides a large, prospective data set of EVT-treated participants with revascularization results and blinded 90-day clinical outcomes. Full methods of the IMS III trial have been reported elsewhere [11]. In brief, 656 participants, aged 18-82 years, were enrolled in this multicenter study. Eligibility criteria included treatment with IV-tPA within $3 \mathrm{~h}$ from symptom onset in participants with moderate to severe stroke (defined as an NIHSS score $\geq 10$ or a score of 8-9 with CT angiographic evidence of an occlusion). Angiographic revascularization grading, according to the mTICI and AOL scales, by a central reader (T.A.T. and D.S.L.) was available for all included participants.

With the aim of studying a homogenous group in the present analysis, we included only participants who had complete occlusions of the internal carotid artery terminus or the proximal middle cerebral artery (M1 and M2) on baseline digital subtraction angiography and who completed EVT within $7 \mathrm{~h}$ after the onset of stroke symptoms. The abilities of the revascularization scales to predict a favorable outcome (defined as a modified Rankin Scale score of 0-2 at 3 months) were compared by receiver operating characteristic (ROC) curves. The optimal thresholds for the predictive accuracy were identified. 


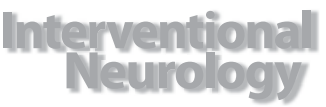

Table 1. Revascularization scores and clinical outcome

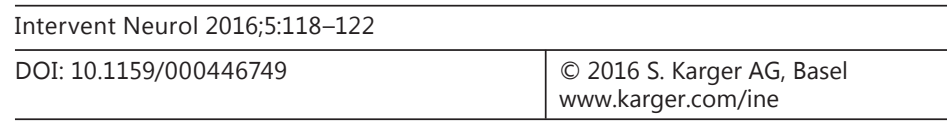

Schmitz et al.: Recanalization and Angiographic Reperfusion Are Both Associated with a Favorable Clinical Outcome in the IMS III Trial

\begin{tabular}{lc}
\hline & Favorable outcome (mRS score of $0-2)$ \\
\hline mTICI score & \\
0 & $1 / 37(2.7 \%)$ \\
1 & $5 / 21(23.8 \%)$ \\
$2 \mathrm{a}$ & $25 / 82(30.5 \%)$ \\
$2 \mathrm{~b}$ & $43 / 94(45.7 \%)$ \\
3 & $5 / 6(83.3 \%)$ \\
\hline AOL score & \\
0 & $1 / 33(3.0 \%)$ \\
1 & $2 / 12(16.7 \%)$ \\
2 & $11 / 44(25.0 \%)$ \\
3 & $65 / 151(43.0 \%)$ \\
\hline
\end{tabular}

\section{Results}

Overall, 240 (57\%) of 423 participants with EVT completed within $7 \mathrm{~h}$ from symptom onset and with complete anterior circulation occlusions were included in our analysis. Among these 240 participants, 61 received only intra-arterial rtPA, 82 MERCI, 40 Penumbra aspiration, 5 stent retrievers, and 52 other or at least two different devices in the same participant. A total of 79 participants (33\%) achieved a favorable outcome. Higher scores of mTICI and AOL were both significantly associated with an increased likelihood of a favorable outcome (Cochran-Armitage trend test $\mathrm{p}<0.0001$; table 1). Thus, among participants with mTICI $3,83.3 \%$ achieved a favorable outcome, whereas only $2.7 \%$ of the participants with mTICI 0 had a favorable outcome (table 1). Similarly, a favorable outcome was reached by $43 \%$ of participants with AOL 3 compared to 3\% of participants with AOL 0 (table 1). Both mTICI reperfusion and AOL recanalization predicted a favorable outcome $(p<0.01)$ with comparable accuracy; the area under the ROC curve was 0.69 (95\% CI, 0.63-0.76) for mTICI and $0.66(95 \% \mathrm{CI}, 0.61-0.72)$ for AOL (fig. 1,2$)$. The optimal thresholds for predictive accuracy were mTICI $2 \mathrm{~b}$ and 3 (sensitivity 61\%, specificity 68\%) and AOL 3 (sensitivity 82\%, specificity $47 \%)$.

\section{Discussion}

Our results, based on data from the IMS III trial, suggest that recanalization and reperfusion predict a favorable outcome with comparable accuracy in ischemic stroke patients who receive EVT. Our analysis is limited to devices used in the IMS III trial, in which only 45 of 240 participants were treated with modern devices, consisting of either Penumbra aspiration or stent retrievers. Modern devices, predominantly used in more recent positive trials, have more rapid and more frequent reperfusion than older clot retrieval devices and may show an even higher correlation between recanalization and angiographic reperfusion. However, recanalization rates relative to reperfusion rates in these recent trials have yet to be reported.

The angiographic threshold that most accurately correlates to a favorable outcome has important clinical relevance. The question of how aggressively to pursue a perfect TICI 3 result remains unanswered. It may be that a TICI $2 \mathrm{~b}$ goal, followed by allowing time for spontaneous recanalization of distal emboli aided by more proximal revascularization and/or concurrently active IV rtPA, should be the goal, as opposed to further EVT and the risk of 
Fig. 1. Unadjusted ROC curve: prediction of a favorable outcome by the mTICI score.
Fig. 2. Unadjusted ROC curve: prediction of a favorable outcome by the AOL score.
Schmitz et al.: Recanalization and Angiographic Reperfusion Are Both Associated with a Favorable Clinical Outcome in the IMS III Trial
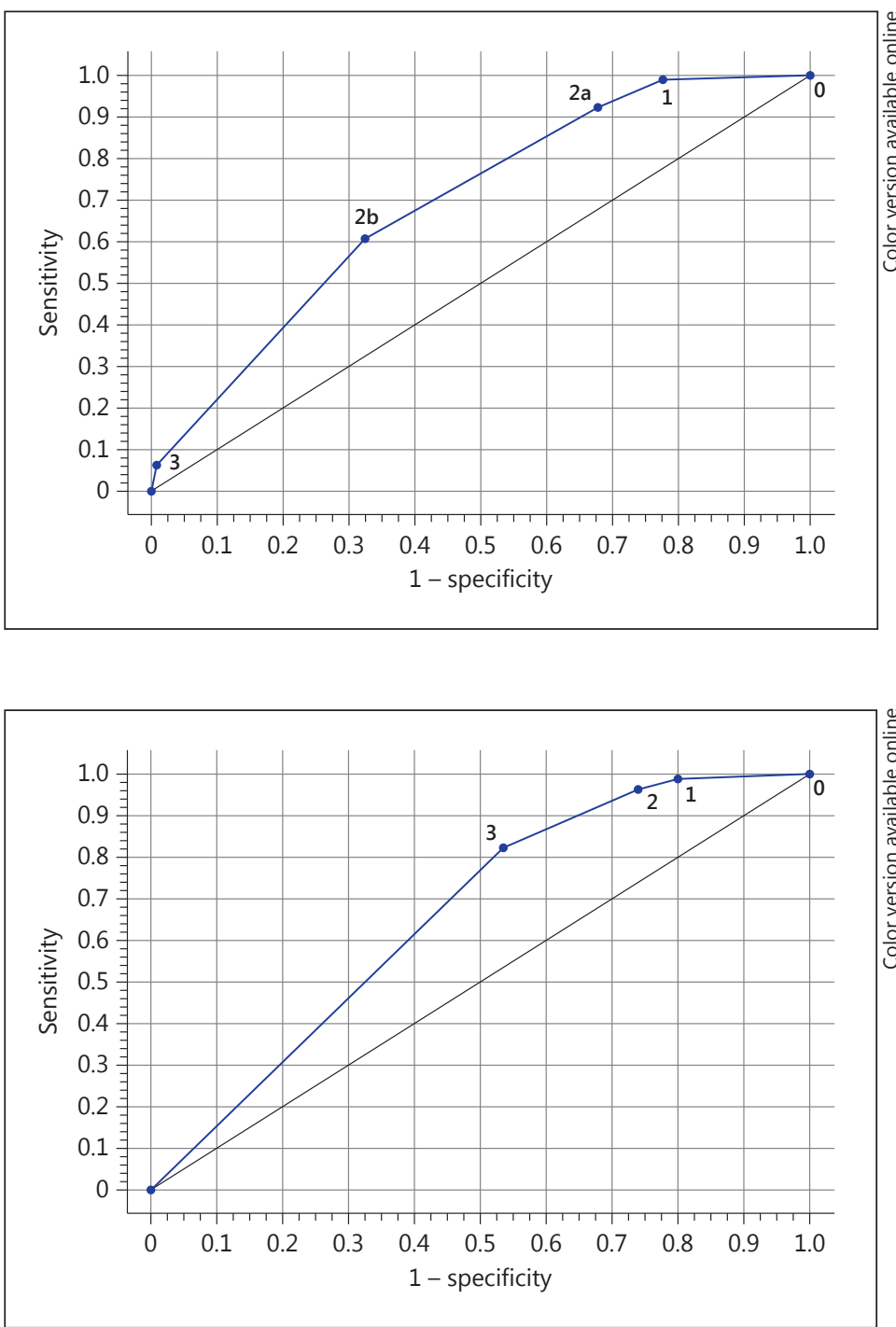

intervening in more distal vasculature. Thus, additional results on the association between angiographic revascularization and clinical outcome from the most recent EVT trials are warranted to further improve the knowledge about the optimal revascularization scores for a favorable outcome. As devices evolve, continued reporting of both recanalization and reperfusion, as well as of new distal embolization, as angiographic outcome measures in studies of EVT-treated participants is recommended.

\section{Disclosure Statement}

S.D.Y.: consultant for Genentech (PRISMS Trial Steering Committee). D.S.L.: consultant roles for Imaging and Angiography Core Labs for Medtronic and Stryker. A.V.: CTSA 8 UL1 TR000077-05 KL2 grant and grant support from Genentech, Inc., for the Imaging Core Laboratory of Study of the Efficacy and Safety of Alteplase in Patients with Mild Stroke (PRISMS) trial. J.P.B.: research monies to the Department of Neurology and Rehabilitation Medicine from Genentech for the PRISMS trial; study medication from Genentech for the IMS III trial, and study catheters supplied during Protocol Versions 1-3 by Concentric, Inc., EKOS Corp., and Cordis 
Schmitz et al.: Recanalization and Angiographic Reperfusion Are Both Associated with a Favorable Clinical Outcome in the IMS III Trial

Neurovascular. P.K.: the University of Cincinnati receives payment for P.K.'s research efforts from Genentech (PRISMS Lead PI), Penumbra (THERAPY Neurology Lead PI), and Biogen (DSMB member). She also received payments from medicolegal consultations, Grand Rounds Experts (online clinical consultations), and UpToDate, Inc. (royalties for online publication).

\section{References}

1 Rha JH, Saver JL: The impact of recanalization on ischemic stroke outcome: a meta-analysis. Stroke 2007;38: 967-973.

2 Zaidat 00, Yoo AJ, Khatri P, et al: Recommendations on angiographic revascularization grading standards for acute ischemic stroke: a consensus statement. Stroke 2013;44:2650-2663.

3 Khatri P, Neff J, Broderick JP, et al: Revascularization end points in stroke interventional trials: recanalization versus reperfusion in IMS-I. Stroke 2005;36:2400-2403.

4 Berkhemer OA, Fransen PS, Beumer D, et al: A randomized trial of intraarterial treatment for acute ischemic stroke. N Engl J Med 2015;372:11-20.

5 Campbell BC, Mitchell PJ, Kleinig TJ, et al: Endovascular therapy for ischemic stroke with perfusion-imaging selection. N Engl J Med 2015;372:1009-1018.

6 Goyal M, Demchuk AM, Menon BK, et al: Randomized assessment of rapid endovascular treatment of ischemic stroke. N Engl J Med 2015;372:1019-1030.

7 Jovin TG, Chamorro A, Cobo E, et al: Thrombectomy within $8 \mathrm{~h}$ after symptom onset in ischemic stroke. $\mathrm{N}$ Engl J Med 2015;372:2296-2306.

8 Saver JL, Goyal M, Bonafe A, et al: Stent-retriever thrombectomy after intravenous t-PA vs t-PA alone in stroke. N Engl J Med 2015;372:2285-2295.

9 Broderick JP, Palesch YY, Demchuk AM, et al: Endovascular therapy after intravenous t-PA versus t-PA alone for stroke. N Engl J Med 2013;368:893-903.

10 Demchuk AM, Goyal M, Yeatts SD, et al: Recanalization and clinical outcome of occlusion sites at baseline CT angiography in the Interventional Management of Stroke III trial. Radiology 2014;273:202-210.

11 Khatri P, Hill MD, Palesch YY, et al: Methodology of the Interventional Management of Stroke III trial. Int J Stroke 2008;3:130-137. 